\title{
THE
}

\section{Comparative Effectiveness and Safety of Prasugrel versus Ticagrelor following Percutaneous Coronary Intervention: An Observational Study}

Nicholas Belviso

University of Rhode Island

D. Aronow

Richard Wyss

Marilyn Barbour

University of Rhode Island, mbarbour@uri.edu

Yichi Zhang

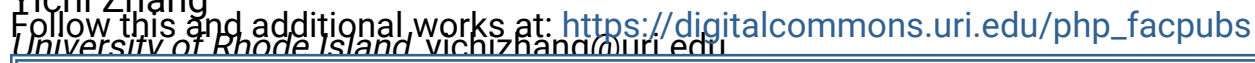

The University of Rhode Island Faculty have made this article openly available. Please let us know how Open Access to this research benefits you.

This is a pre-publication author manuscript of the final, published article.

Terms of Use

This article is made available under the terms and conditions applicable towards Open Access Policy Articles, as set forth in our Terms of Use.

\section{Citation/Publisher Attribution}

Belviso, N., Aronow, H.D., Wyss, R., Barbour, M., Zhang, Y., Wen, X. and Kogut, S. (2021), Comparative Effectiveness and Safety of Prasugrel versus Ticagrelor following Percutaneous Coronary Intervention: An Observational Study. Pharmacotherapy. Accepted Author Manuscript. https://doi.org/10.1002/phar.2530 Available at: https://doi.org/10.1002/phar.2530 
Authors

Nicholas Belviso, D. Aronow, Richard Wyss, Marilyn Barbour, Yichi Zhang, Xuerong Wen, and Stephen Kogut

This article is available at DigitalCommons@URI: https://digitalcommons.uri.edu/php_facpubs/205 


\section{PHARMACOTHERAPY}

\section{Comparative Effectiveness and Safety of Prasugrel versus Ticagrelor following Percutaneous Coronary Intervention: An Observational Study}

\begin{tabular}{|c|c|}
\hline Journal: & Pharmacotherapy \\
\hline Manuscript ID & $12-20-0746 . R 1$ \\
\hline Manuscript Type: & Original Research Article \\
\hline $\begin{array}{r}\text { Date Submitted by the } \\
\text { Author: }\end{array}$ & $\mathrm{n} / \mathrm{a}$ \\
\hline Complete List of Authors: & $\begin{array}{l}\text { Belviso, Nicholas; University of Rhode Island, Pharmacy Practice } \\
\text { Aronow, Herbert; Brown University Warren Alpert Medical School, } \\
\text { Interventional Cardiology; Lifespan Health System, Cardiovascular } \\
\text { Institute } \\
\text { Wyss, Richard; Harvard Medical School, Division of } \\
\text { Pharmacoepidemiology and Pharmacoeconomics, Brigham and Women's } \\
\text { Hospital } \\
\text { Barbour, Marilyn; University of Rhode Island - College of Pharmacy, } \\
\text { Pharmacy Practice } \\
\text { Zhang, Yichi; University of Rhode Island College of Engineering, } \\
\text { Department of Computer Science and Statistics } \\
\text { Wen, Xuerong; University of Rhode Island - College of Pharmacy, } \\
\text { Pharmacy Practice } \\
\text { Kogut, Stephen; University of Rhode Island - College of Pharmacy, } \\
\text { Department of Pharmacy Practice }\end{array}$ \\
\hline Keywords: & $\begin{array}{l}\text { Antiplatelets, Drug safety, Epidemiology, Evidence-based medicine, } \\
\text { Outcomes }\end{array}$ \\
\hline Abstract: & $\begin{array}{l}\text { Background: Observational studies comparing ticagrelor and prasugrel in } \\
\text { this setting have yielded contradictory results but often do not consider } \\
\text { differential censoring (e.g., for treatment switching or insurance } \\
\text { disenrollment)or confounding by time dependent factors. } \\
\text { Objective: Our objective was to conduct a comparative effectiveness and } \\
\text { safety analysis of ticagrelor and prasugrel in patients who underwent PCI } \\
\text { after being hospitalized for an acute coronary syndrome. } \\
\text { Methods: This study used the Optum's de-identified Clinformatics } ® \text { Data } \\
\text { Mart Database and included patients aged } 18 \text { years or older with an } \\
\text { index hospital admission between May } 2012 \text { and December } 2015 \text {, a } \\
\text { diagnosis of acute coronary syndrome managed with percutaneous } \\
\text { coronary intervention, and treatment with either ticagrelor or prasugrel. } \\
\text { The primary composite outcome was defined as the first occurrence all- } \\
\text { cause death, myocardial infarction, or ischemic stroke. The secondary } \\
\text { composite outcome included the first occurrence of gastrointestinal } \\
\text { bleed, intracranial hemorrhage, or other major bleeds requiring } \\
\text { hospitalization. Weighted Cox proportional hazard models and robust } \\
\text { variance estimation were implemented to adjust for baseline } \\
\text { comorbidities, time-varying exposure, time-dependent confounders, and } \\
\text { differential censoring. } \\
\text { Results: Included in the analysis were 2,559 patients initiated on }\end{array}$ \\
\hline
\end{tabular}


ticagrelor and 4,456 patients initiated on prasugrel following PCI. Patients initiated on ticagrelor were $10 \%$ more likely to have eligibility disenrollment (Ticagrelor: $57 \%$, Prasugrel: $47 \%, \mathrm{P}<.01$ ) and 7 percentage-points more likely to switch medication (Ticagrelor: $35 \%$, Prasugrel: $28 \%, \mathrm{P}<.01$ ). After adjusting for multiple factors, including time-varying exposure, and censoring imbalance, ticagrelor use was associated with a higher risk of all-cause death, MI, or stroke when compared to prasugrel (HR: $1.33 ; 95 \% \mathrm{CI}: 1.04-1.68$ ). Similarly, ticagrelor was associated with a higher risk in bleeding events when compared with prasugrel (HR: $1.61 ; 95 \% \mathrm{CI}: 1.19-2.17$ ).

Conclusion: When compared with ticagrelor, prasugrel use following PCI for ACS was associated with a lower risk of death, MI, or stroke. as well as with a reduced risk of major bleeding.

\section{SCHOLARONE Manuscripts}




\section{Title: Comparative Effectiveness and Safety of Prasugrel versus Ticagrelor following Percutaneous Coronary Intervention: An Observational Study}

Nicholas Belviso, PharmD MBA ${ }^{1}$; Herbert D. Aronow, MD MPH ${ }^{2,3}$; Richard Wyss, $\mathrm{PhD}^{4}$; Marilyn Barbour, PharmD FCCP${ }^{1}$; Yichi Zhang, $\mathrm{PhD}^{5}$; Xuerong Wen, $\mathrm{PhD}$, $\mathrm{MPH}, \mathrm{MS}^{1 *}$, Stephen Kogut, PhD MBA ${ }^{1 *}$

${ }^{1}$ College of Pharmacy, University of Rhode Island, Kingston, RI

${ }^{2}$ Lifespan Cardiovascular Institute, Providence, RI

${ }^{3}$ Warren Alpert Medical Scholl of Brown University, Providence, RI

${ }^{4}$ Division of Pharmacoepidemiology and Pharmacoeconomics, Brigham and Women's Hospital, Harvard Medical School

${ }^{5}$ Department of Computer Science and Statistics, University of Rhode Island, Kingston, RI

*These two senior authors contributed equally to this project.

Keywords: Comparative Effectiveness and Safety, Prasugrel, Ticagrelor, PCI

Word count: 2973 


\begin{abstract}
Background: Observational studies comparing ticagrelor and prasugrel in this setting have yielded contradictory results but often do not consider differential censoring (e.g., for treatment switching or insurance disenrollment)or confounding by time dependent factors.
\end{abstract}

Objective: Our objective was to conduct a comparative effectiveness and safety analysis of ticagrelor and prasugrel in patients who underwent PCI after being hospitalized for an acute coronary syndrome.

Methods: This study used the Optum's de-identified Clinformatics ${ }^{\circledR}$ Data Mart Database and included patients aged 18 years or older with an index hospital admission between May 2012 and December 2015, a diagnosis of acute coronary syndrome managed with percutaneous coronary intervention, and treatment with either ticagrelor or prasugrel. The primary composite outcome was defined as the first occurrence allcause death, myocardial infarction, or ischemic stroke. The secondary composite outcome included the first occurrence of gastrointestinal bleed, intracranial hemorrhage, or other major bleeds requiring hospitalization. Weighted Cox proportional hazard models and robust variance estimation were implemented to adjust for baseline comorbidities, time-varying exposure, time-dependent confounders, and differential censoring.

Results: Included in the analysis were 2,559 patients initiated on ticagrelor and 4,456 patients initiated on prasugrel following PCI. Patients initiated on ticagrelor were 10\% more likely to have eligibility disenrollment (Ticagrelor: 57\%, Prasugrel: 47\%, P<.01) and 7 percentage-points more likely to switch medication (Ticagrelor: 35\%, Prasugrel: 
$28 \%, \mathrm{P}<.01)$. After adjusting for multiple factors, including time-varying exposure, and censoring imbalance, ticagrelor use was associated with a higher risk of all-cause death, MI, or stroke when compared to prasugrel (HR: 1.33 ; 95\%CI: 1.04-1.68). Similarly, ticagrelor was associated with a higher risk in bleeding events when compared with prasugrel (HR: $1.61 ; 95 \%$ CI: 1.19-2.17).

Conclusion: When compared with ticagrelor, prasugrel use following PCI for ACS was associated with a lower risk of death, MI, or stroke. as well as with a reduced risk of major bleeding. 


\section{INTRODUCTION}

Dual antiplatelet therapy (DAPT), with aspirin and a $\mathrm{P}_{2} \mathrm{Y}_{12}$ agent, reduces the risk of subsequent ischemic events and is a mainstay in treatment in patients with acute coronary syndrome undergoing percutaneous coronary intervention (PCI) ${ }^{1-3}$ In the TRITON-TIMI-38 trial, less ischemia but more bleeding occurred in the prasugrel than clopidogrel group. In contrast, in the PLATO trial comparing ticagrelor and clopidogrel, both agents reduce CV death, MI, and stroke by the same magnitude and both agent increase non-CABG TIMI major bleeding by the same magnitude.. The Intracoronary Stenting and Antithrombotic Regimen: Rapid Early Action for Coronary Treatment (ISAR-REACT) 5 trial, a randomized, open-label study evaluating ticagrelor versus prasugrel in patients with acute coronary syndrome (ACS) in whom an invasive evaluation was planned, found that patients receiving ticagrelor had a significantly higher incidence of death, myocardial infarction, or stroke but similar rates of major bleeding over 1 year of follow up. ${ }^{4}$ Observational studies comparing ticagrelor and prasugrel in this setting have yielded contradictory results, ${ }^{5-9}$ but have not taken into account the possibility of differential censoring (e.g., for treatment switching or insurance disenrollment), nor of confounding by time dependent factors, both of which can affect study outcomes. ${ }^{10}$

We conducted a comparative effectiveness and safety analysis of ticagrelor and prasugrel in patients who underwent PCI for an acute coronary syndrome. We implemented marginal structural models and employed inverse probability censoring weighting to adjust for post-treatment selection bias caused by imbalance in treatment 
switching and insurance disenrollment between comparison groups. We hypothesized that after adequate adjustment for confounding and selection bias, a real-world comparative effectiveness and safety study would yield results similar to those observed in the ISAR-REACT 5 trial.

\section{METHODS}

\section{Data Source}

We utilized Optum's de-identified Clinformatics ${ }^{\circledR}$ Data Mart Database (Optum Inc., Eden Prairie, MN), a large, nationwide, managed care, administrative claims dataset comprised of longitudinal medical billing information in the United States. Insurance claims for all pharmacy, inpatient, and outpatient services are included for the enrolled 13 million yearly-members. ${ }^{11}$ This project was designated as "research not involving human subjects" by the University of Rhode Island Institutional Review Board, as all data were de-identified prior to analyses.

\section{Study Cohort}

Patients aged 18 years and older hospitalized between May 2012 and September 2015 with a diagnosis of ACS managed with PCI and treated with either prasugrel or ticagrelor were included. This period was selected to align with the FDA approval of ticagrelor in July 2011, allowing for delayed acceptance into insurance formularies. ACS was identified using the International Classification of Diseases, Ninth Revision, Clinical Modification (ICD-9-CM) codes (ICD-9-CM: 410.x [acute myocardial infarction] and 411.x [other acute and subacute forms of ischemic heart disease]). ${ }^{12}$ 
PCI was identified by ICD-9-CM and Current Procedural Terminology (CPT-4) procedure codes (ICD-9-CM: 00.66, 36.01, 36.02, 36.05, 36.06, 36.07, and 36.09; CPT-4: 92980, 92981, 92982, 92984, 92920, 92921, 92924, 92925, 92928, 92929, 92933, 92934, 92937, 92938, 92941, 92943, 92944, 92973) occurring during the index hospitalization. ${ }^{9}$ At least one pharmacy prescription claim for either ticagrelor or prasugrel within 14 days of discharge was required. ${ }^{9}$ Patients with prior dispensing of ticagrelor or prasugrel, history of stroke, fibrinolytic therapy within one day of hospitalization, prior dispensing of oral anticoagulants, or dispensing of strong Cytochrome P-450 3A inhibitors/inducers identified during the baseline period were excluded. ${ }^{4,13}$ Additionally, patients with claims for more than one or any non-study antiplatelet agents during the 14-day initiation window were excluded.

\section{Outcomes Assessments}

The primary composite outcome was defined as the first occurrence of one of any of the following: all-cause death, myocardial infarction, or ischemic stroke. All-cause death was identified utilizing the Social Security Administration's Death Master File. Since only the month and year of death were included in these data, a day of death was randomly assigned within each month for death events occurring after hospital discharge. Myocardial infarction and stroke were identified by ICD-9-CM diagnosis codes (MI: 410-412 [excluding 410.x2]; Stroke: 430-434, 436) occurring during hospitalization. ${ }^{14,15}$ The secondary composite outcome was defined as the first occurrence of one of the following: gastrointestinal bleed, intracranial hemorrhage, or other major bleeding requiring hospitalization. ${ }^{16}$ Patients were followed from index 
hospitalization discharge until primary endpoint occurrence, loss of insurance eligibility, or for 365 days; whichever occurred first.

\section{Censoring Assessment}

Censoring events included insurance disenrollment, treatment switching, treatment discontinuation, or end of data window on September 30, 2015. Insurance disenrollment was identified by eligibility end or a gap of 30 days or more in insurance enrollment. Treatment switching was classified by the discontinuation of the initially prescribed antiplatelet therapy and initiation of an alternative medication, as identified via prescription pharmacy claims. Patients who switched to ticagrelor or prasugrel were not censored at switch date consistent with the time-varying exposure analysis described below. However, patients that switched to clopidogrel were censored. Therapy discontinuation was defined as a greater than 45 days of gap between prescription end of supply and refill. Since treatment duration of 6 to 12 months is recommended in this clinical setting, gaps greater than 45 days occurring after 6-months of follow-up were not evaluated. ${ }^{17}$

\section{Covariate Assessment}

Baseline covariates assessed during the 6-month baseline window included: age, sex, hypertension, tobacco use, hyperlipidemia, major bleeding, peripheral vascular disease, chronic kidney disease, dialysis, anemia, chronic obstructive pulmonary disease, previous percutaneous transluminal angioplasty, previous coronary artery bypass graft, congestive heart failure, atrial fibrillation, and beta-blocker, diuretic, statin, proton- 
pump inhibitor, or diabetes medication use. These are time-fixed potential confounding factors specified by previous studies. ${ }^{17,18} \mathrm{~A}$ time variable was generated for each person-time interval when the exposure was assessed. Angina, prior interval treatment, and interval time were included as time-dependent covariates assessed during each month of follow-up.

\section{Statistical Analysis}

Categorical variables are presented as frequencies (\%) and compared using chi-square tests. Continuous variables are presented as mean \pm standard deviation (SD) and were compared between two drug groups using student t-test. Marginal-structural models (MSM) were fit using inverse probability weights (IPW) to adjust for confounding with time-dependent variables. ${ }^{19,20}$ Possible violations of positivity and misspecification were assessed in all models by inspecting the estimated stabilized weight distribution to check for extreme values and to confirm that the mean was approximately equal to one. ${ }^{21}$ The proportional hazards assumption was assessed graphically by examining the IPW log cumulative hazard function estimates to ensure that the hazard curves remained parallel over time.

\section{Time-Dependent Exposure (TD)}

Weighted Cox proportional hazard models and robust variance estimation were implemented to adjust for baseline and time-dependent confounders. ${ }^{22,23}$ The robust variance estimator was required to account for the additional variability introduced by estimating the IPWs. The time-dependent weights were constructed to adjust for fixed 
baseline and time-varying confounding factors. Exposure was assessed at monthly intervals of prescription dispensing for each patient following initial assignment and continued until loss of eligibility, switching to clopidogrel, treatment discontinuation, event occurrence, or study end. Weights for each person-time interval were created by the ratio of the probability that each patient received their observed treatment conditional on time, and past treatment divided by the probability that the patient received the observed treatment given time, past treatment, baseline covariates, and prognostic (time-dependent) factors..$^{24}$

\section{Censoring Weighting $(\mathrm{CW})$}

The censor-weighting accounted for possible informative censoring (e.g., insurance disenrollment) due to measured confounding factors. These weights were estimated as the ratio of the probability of remaining enrolled in the insurance program for 12-months following index hospitalization given time of the exposure assessed, prior treatment divided by the conditional probability of remaining enrolled given time, prior treatment, baseline variables, and time-dependent confounding factors. ${ }^{24,25}$ The time-dependent exposure and censoring weights were multiplied together for each person-time interval of follow-up for each patient.

\section{Sensitivity Analyses}

Intention-To-Treat Analysis (ITT)

A conventional ITT analysis was conducted to better enable comparison with the approaches used in previously published observational studies. Patients were censored 
if loss of follow-up due to insurance disenrollment but were not censored based on treatment discontinuation, adherence, or switching. The IPWs were derived by a ratio of the marginal probability of exposure and the probability of exposure given baseline covariates. ${ }^{26}$ The weights were used to create a pseudopopulation in which the measured covariates and treatment assignment were independent of each other. No adjustments were made for time-dependent confounding factors. We hypothesized that not adjusting for dropout or switching imbalances between exposure groups would bias results toward the null.

\section{Clopidogrel Naïve Population}

To determine if clopidogrel exposure during baseline period was associated with the study outcomes, we narrowed the population to antiplatelet-naïve patients by excluding those with clopidogrel exposure during the baseline period. While the ISAR-REACT 5 trial did not exclude patients with a history of clopidogrel use, we hypothesized that the results would be similar for a population that was naïve to P2 $\mathrm{Y}_{12}$-antiplatelet therapy.

\section{RESULTS}

\section{Study Population}

There were 91,682 individuals admitted with an ACS who underwent PCI during the study period. Of these, 71,287 had 6-months of insurance eligibility prior to index hospitalization. After applying the exclusion criteria, there were 2,559 initiated on 
ticagrelor and 4,456 initiated on prasugrel following PCI who were included in this analysis (Figure 1).

The ticagrelor group had significantly higher incidences of comorbidities at baseline as compared with those who initiated prasugrel (Table 1). The ticagrelor group was older, had higher rates of hypertension, chronic kidney disease, anemia, chronic obstructive pulmonary disease, congestive heart failure, and atrial fibrillation. Patients initiated on ticagrelor were $10 \%$ higher on eligibility disenrollment and $7 \%$ higher on medication switching (absolute differences in Table 2). The ticagrelor group had a 2 percentagepoint higher use of clopidogrel during baseline compared to the prasugrel group (absolute differences in Table 2). Treatment discontinuation was balanced between groups.

\section{Comparative Effectiveness Results}

In the unadjusted analysis, the composite outcome, death, myocardial infarction, or ischemic stroke, occurred numerically more often in the ticagrelor group (Table 3). The rates of gastrointestinal bleeding, intracranial hemorrhage, and other major bleeding requiring hospitalization were also numerically greater in the ticagrelor group, however neither difference in composite endpoint rates was statistically significant. After adjusting for multiple confounding factors, time-varying exposure, and censoring imbalance, ticagrelor was associated with a higher risk of the composite all-cause death, MI, or stroke, when compared to prasugrel (HR: $1.33 ; 95 \% \mathrm{CI}: 1.04-1.68 ; \mathrm{p}=0.02$ )

(Table 4). Similarly, ticagrelor was also associated with a higher risk of bleeding events 
(HR: $1.61 ; 95 \%$ CI: $1.19-2.17 ; \mathrm{p}<0.01$ ). Figures 2 and 3 show the survival curves for outcomes in the IPW estimated pseudopopulation.

\section{Sensitivity Analysis Results}

Intention-To-Treat Analysis (ITT)

The conventional ITT analysis that did not adjust for the disenrollment or switching imbalance between groups derived estimates in the opposite direction. With the ITT approach, ticagrelor was associated with numerically fewer all-cause death, MI, and stroke events (HR: 0.78; 95\%CI: 0.58-1.06; $\mathrm{p}=0.11$ ) and numerically fewer bleeding events (HR: $0.84 ; 95 \% \mathrm{CI}: 0.58-1.20, \mathrm{p}=0.33$ ), but these differences were nonsignificant.

\section{Clopidogrel Naïve Population}

Results for the sensitivity analyses that excluded patients dispensed clopidogrel during baseline were similar to those from the ISAR-REACT 5 study population, where prior clopidogrel use was allowed. The TD-CW method for ischemic (HR: 1.28; 95\%CI: 0.99-1.66; $\mathrm{p}=0.06)$ and bleeding (HR: 1.63; 95\%CI: 1.19-2.23, $\mathrm{p}<0.01)$ composite outcomes remained consistent. Similarly, ITT results for the primary composite outcome (HR: $0.81 ; 95 \% \mathrm{CI}: 0.59-1.11 ; \mathrm{p}=0.19)$ and secondary composite outcome (HR: $1.05 ; 95 \% \mathrm{CI}: 0.73-1.51 ; \mathrm{p}=0.79$ ) were similar in direction although not significantly different.

\section{DISCUSSION}


In this real-world study using a national claims dataset we compared the incidence of composite ischemic and bleeding events between hospitalized patients prescribed ticagrelor or prasugrel after PCI for an ACS. Two analytic methods were applied. In TD-CW analyses with Marginal Structural Models, ticagrelor was associated with a 33\% increased hazard of all-cause death, MI, or stroke, as well as a $61 \%$ greater hazard for ICH, GI bleed, or major hemorrhage. However, in traditional ITT analyses there were no significant differences in ischemic or bleeding outcomes between treatments.

Our findings from the TD-CW analysis were consistent with the ISAR-REACT 5 trial which found that prasugrel was associated with a lower risk of ischemic and bleeding events in patients treated for ACS. While treatment guidelines recommend prasugrel or ticagrelor equally following PCI, many clinical and non-clinical factors can influence treatment selection, potentially explaining the observed imbalances in disenrollment and antiplatelet switching. ${ }^{17,27}$ While observational studies can adjust for baseline imbalances between groups, post-exposure events are not often evaluated. We implemented a time-dependent exposure and censor weighted model to adjust for the censoring imbalances identified in Table 2. In order to replicate/mimic findings from the RCT, we need to conduct a study in which adherence is similar. To do this, we need to account for time-varying confounding and censoring to get an accurate 'on treatment' effect. Thus, we applied methods for time-dependent confounding, drug switching and discontinuation to account for differences in adherence/discontinuation between the 
real-world data and RCT. Accounting for these factors within the observational cohort resulted in dropout and treatment switching rates that were similar to the ISAR-REACT 5 trial. The results from this analysis were in the same direction as the trial results.

The observational literature regarding outcomes of antiplatelet use have conflicting results. ${ }^{5-9,18,28}$ Differences in how study designs address variability in follow-up time, inclusion and exclusion criteria, approaches for addressing covariates may lead to divergent conclusions. Using a conventional ITT analytic approach that fails to address censoring events that are imbalanced between treatment groups, and differential censoring can lead to different interpretations of the estimates. Dawwas et al. evaluated the comparative effectiveness and safety of ticagrelor versus prasugrel in patients with ACS. ${ }^{28}$ Their results indicated that ticagrelor was associated with a decreased risk of recurrent nonfatal CVD events (HR: 0.80, 95\%CI: 0.70-0.92), and major bleeding events (HR: 0.54, 95\%CI: 0.41-0.70). In contrast, some observational studies suggested a benefit with prasugrel over ticagrelor. For example, Larmore et al. found that major adverse cardiovascular and major bleeding events were lower at 30-days in the prasugrel-treated group when compared to the ticagrelor-treated group. ${ }^{29}$ However, most observational studies found no significant difference in the incidence of adverse cardiovascular and bleeding events when comparing these agents..$^{7-11}$ Our ITT sensitivity analysis provided results that were consistent with recent observational studies evaluating these agents and that were contrary to the ISAR-REACT 5 trial. Ignoring differential post-assignment imbalances between exposure groups produced results in the opposite direction. The variability of results in observational studies may 
be attributed to different study populations, and most likely unaddressed post-treatment selection bias as none of these studies assessed or compared medication switching or disenrollment rates in comparison groups during study follow-up.

While there was a statistically significant $2 \%$ higher clopidogrel exposure rate in the ticagrelor group during the baseline period, narrowing the population to antiplateletnaïve patients by excluding those with prior clopidogrel exposure produced similar results with a slight loss in power. This suggests that clopidogrel use at baseline period did not impact the estimated treatment effect.

The key difference between the analysis methods lies in adjusting for confounding that results in incomplete adherence to the assigned treatment. It has been noted in clinical trials that loss to follow-up may be affected by clinical or demographical factors occurring after randomization. Our results further confirmed that the imbalance of medication switching or insurance disenrollment between exposures could bias the results. Adjusting for post-assignment factors can help improve the precision of effect estimates. After adjusting for time-varying confounding and treatment in the TD-CW analysis, our findings were consistent with the ISAR-REACT 5 trial where the posttreatment selection bias was addressed.

\section{LIMITATIONS}

First, this study is based on claims data and could be biased by unmeasured confounding factors (e.g., over-the-counter medications, antiplatelet loading-dose, or other details of 
inpatient procedures). Second, a substantial number of patients were excluded for not having an outpatient dispensing of the study treatment within 14-days of index PCI. Subsequent analysis of the 27,049 patients excluded indicated that the majority did not have pharmacy dispensings of the study agents. The 14-day window captured $>83 \%$ of the patients with pharmacy dispensing of the study treatments in the database. We presume that most of the patients with no dispensings of antiplatelet agents following PCI were treated and recorded using a different payment system. Increasing this window to 30 and 90 days did not greatly improve the number of patients identified. However, these effects are thought to be non-differential as we are comparing two drugs from the same drug class. Third sub-codes differentiating between types of myocardial events such as STEMI or NSTEMI were not reliably utilized in the data to differentiate as outcomes. Fourth, our results pertain to patients enrolled in a managed care plan and may not be generalizable to other populations. Additionally, the death file included within this dataset is the Social Security Administration Death Master file. Since 2013 it was no longer mandatory for states to report death events to the Social Security Administration. As such, death events are expected to be non-differentially underreported. ${ }^{30}$ Lastly, there are several key assumptions that must be made to obtain correct causal inferences from the time-varying approaches. We assumed that the measured covariates, including baseline and time-varying factors, were sufficient to adjust for both confounding and post-treatment selection bias. This assumption is not testable in retrospective observational studies; however, we relied on comprehensive literature review and clinical expertise to bolster this assumption. While many of the covariates were selected to mimic previous clinical trials, information regarding 
gastrointestinal disorders or abnormalities may be factors of interest for future studies. We also assumed that the models implemented were suitably specified, including the MSM comparing average treatment effects conditional on time-varying exposure, baseline covariates, and time-varying confounders. While these assumptions are not testable, we fit the same covariates in all models to make these results comparable.

\section{CONCLUSION}

After adjusting for confounding factors, time-varying exposure, and censoring imbalance using marginal structural models with TD-CW, results from our observational data mirrored those from a contemporary randomized controlled trial asking the same question; application of such statistical methods may augment future comparative effectiveness and safety analyses using observational data. 


\section{REFERENCES}

1. Wiviott SD, Braunwald E, McCabe CH, et al. Prasugrel versus clopidogrel in patients with acute coronary syndromes. N Engl J Med. 2007;357(20):20012015. doi:10.1056/NEJMoa0706482

2. Wallentin L, Becker RC, Budaj A, et al. Ticagrelor versus clopidogrel in patients with acute coronary syndromes. $N$ Engl J Med. 2009;361(11):1045-1057. doi:10.1056/NEJMoa0904327

3. Piccolo R, Windecker S. Dual Antiplatelet Therapy in Percutaneous Coronary Intervention: A Tale of 2 Decades With New Perspectives in the Era of NewGeneration Drug-Eluting Stents. Circ Cardiovasc Interv. 2016;9(2):e003587. doi:10.1161/CIRCINTERVENTIONS.116.003587

4. Schüpke S, Neumann F-J, Menichelli M, et al. Ticagrelor or Prasugrel in Patients with Acute Coronary Syndromes. New England Journal of Medicine. 2019;381(16):1524-1534. doi:10.1056/NEJMoa1908973

5. Song C, Sukul D, Seth M, et al. Ninety-Day Readmission and Long-Term Mortality in Medicare Patients ( $\geq 65$ Years) Treated With Ticagrelor Versus Prasugrel After Percutaneous Coronary Intervention (from the Blue Cross Blue Shield of Michigan Cardiovascular Consortium). Am J Cardiol. 2017;120(11):1926-1932. doi:10.1016/j.amjcard.2017.08.009

6. Yudi MB, Clark DJ, Farouque O, et al. Clopidogrel, prasugrel or ticagrelor in patients with acute coronary syndromes undergoing percutaneous coronary intervention. Intern Med J. 2016;46(5):559-565. doi:10.1111/imj.13041

7. Alexopoulos D, Xanthopoulou I, Deftereos S, et al. Contemporary antiplatelet treatment in acute coronary syndrome patients undergoing percutaneous coronary intervention: 1-year outcomes from the GReek AntiPlatElet (GRAPE) Registry. $J$ Thromb Haemost. 2016;14(6):1146-1154. doi:10.1111/jth.13316

8. Coons JC, Iasella CJ, Chanas T, et al. Comparative Effectiveness and Safety Analysis of Dual Antiplatelet Therapies Within an Integrated Delivery System. Ann Pharmacother. 2017;51(8):649-655. doi:10.1177/1060028017706977

9. Kim K, Lee TA, Ardati AK, DiDomenico RJ, Touchette DR, Walton SM. Comparative Effectiveness of Oral Antiplatelet Agents in Patients with Acute Coronary Syndrome. Pharmacotherapy. 2017;37(8):877-887.

doi:10.1002/phar.1961

10. Leung KM, Elashoff RM, Afifi AA. Censoring issues in survival analysis. Annu Rev Public Health. 1997;18:83-104. doi:10.1146/annurev.publhealth.18.1.83

11. Health Services and Innovation Company. Accessed April 16, 2018. https://www.optum.com/ 
12. Varas-Lorenzo C, Castellsague J, Stang MR, Tomas L, Aguado J, PerezGutthann S. Positive predictive value of ICD-9 codes 410 and 411 in the identification of cases of acute coronary syndromes in the Saskatchewan Hospital automated database. Pharmacoepidemiol Drug Saf. 2008;17(8):842-852. doi: $10.1002 /$ pds. 1619

13. What are some common medications classified as weak, moderate and strong inhibitors of CYP3A4? Accessed December 10, 2019. https://www.ebmconsult.com/articles/medications-inhibitors-cyp3a4-enzyme

14. Wahl PM, Rodgers K, Schneeweiss S, et al. Validation of claims-based diagnostic and procedure codes for cardiovascular and gastrointestinal serious adverse events in a commercially-insured population. Pharmacoepidemiol Drug Saf. 2010;19(6):596-603. doi:10.1002/pds.1924

15. Thigpen JL, Dillon C, Forster KB, et al. Validity of international classification of disease codes to identify ischemic stroke and intracranial hemorrhage among individuals with associated diagnosis of atrial fibrillation. Circ Cardiovasc Qual Outcomes. 2015;8(1):8-14. doi:10.1161/CIRCOUTCOMES.113.000371

16. Cunningham A, Stein CM, Chung CP, Daugherty JR, Smalley WE, Ray WA. An automated database case definition for serious bleeding related to oral anticoagulant use. Pharmacoepidemiol Drug Saf. 2011;20(6):560-566. doi: $10.1002 /$ pds.2109

17. Levine GN, Bates ER, Bittl JA, et al. 2016 ACC/AHA Guideline Focused Update on Duration of Dual Antiplatelet Therapy in Patients With Coronary Artery Disease: A Report of the American College of Cardiology/American Heart Association Task Force on Clinical Practice Guidelines. Journal of the American College of Cardiology. 2016;68(10):1082-1115. doi:10.1016/j.jacc.2016.03.513

18. Larmore C, Effron MB, Molife C, et al. "Real-World" Comparison of Prasugrel With Ticagrelor in Patients With Acute Coronary Syndrome Treated With Percutaneous Coronary Intervention in the United States. Catheter Cardiovasc Interv. 2016;88(4):535-544. doi:10.1002/ccd.26279

19. Hernán MA, Hernández-Díaz S, Robins JM. A structural approach to selection bias. Epidemiology. 2004;15(5):615-625.

20. Robins JM, Finkelstein DM. Correcting for noncompliance and dependent censoring in an AIDS Clinical Trial with inverse probability of censoring weighted (IPCW) log-rank tests. Biometrics. 2000;56(3):779-788.

21. Yang S, Eaton CB, Lu J, Lapane KL. Application of marginal structural models in pharmacoepidemiologic studies: a systematic review. Pharmacoepidemiol Drug Saf. 2014;23(6):560-571. doi:10.1002/pds.3569 
22. Austin PC. Variance estimation when using inverse probability of treatment weighting (IPTW) with survival analysis. Stat Med. 2016;35(30):5642-5655. doi:10.1002/sim.7084

23. Austin PC, Stuart EA. Moving towards best practice when using inverse probability of treatment weighting (IPTW) using the propensity score to estimate causal treatment effects in observational studies. Stat Med. 2015;34(28):36613679. doi:10.1002/sim. 6607

24. Hernán MA, Brumback B, Robins JM. Marginal structural models to estimate the causal effect of zidovudine on the survival of HIV-positive men. Epidemiology. 2000;11(5):561-570.

25. Cole SR, Hernán MA. Constructing Inverse Probability Weights for Marginal Structural Models. Am J Epidemiol. 2008;168(6):656-664. doi:10.1093/aje/kwn164

26. Xu S, Ross C, Raebel MA, Shetterly S, Blanchette C, Smith D. Use of stabilized inverse propensity scores as weights to directly estimate relative risk and its confidence intervals. Value Health. 2010;13(2):273-277. doi:10.1111/j.15244733.2009.00671.x

27. Authors/Task Force members, Windecker S, Kolh P, et al. 2014 ESC/EACTS Guidelines on myocardial revascularization: The Task Force on Myocardial Revascularization of the European Society of Cardiology (ESC) and the European Association for Cardio-Thoracic Surgery (EACTS)Developed with the special contribution of the European Association of Percutaneous Cardiovascular Interventions (EAPCI). Eur Heart J. 2014;35(37):2541-2619. doi:10.1093/eurheartj/ehu278

28. Dawwas GK, Dietrich E, Winchester DE, Winterstein AG, Segal R, Park H. Comparative Effectiveness and Safety of Ticagrelor versus Prasugrel in Patients with Acute Coronary Syndrome: A Retrospective Cohort Analysis.

Pharmacotherapy. 2019;39(9):912-920. doi:10.1002/phar.2311

29. Larmore C, Effron MB, Molife C, et al. "Real-World" Comparison of Prasugrel With Ticagrelor in Patients With Acute Coronary Syndrome Treated With Percutaneous Coronary Intervention in the United States. Catheter Cardiovasc Interv. 2016;88(4):535-544. doi:10.1002/ccd.26279

30. Levin MA, Lin H-M, Prabhakar G, McCormick PJ, Egorova NN. Alive or dead: Validity of the Social Security Administration Death Master File after 2011. Health Services Research. 2019;54(1):24-33. doi:10.1111/1475-6773.13069 
Figure 1. Study Population

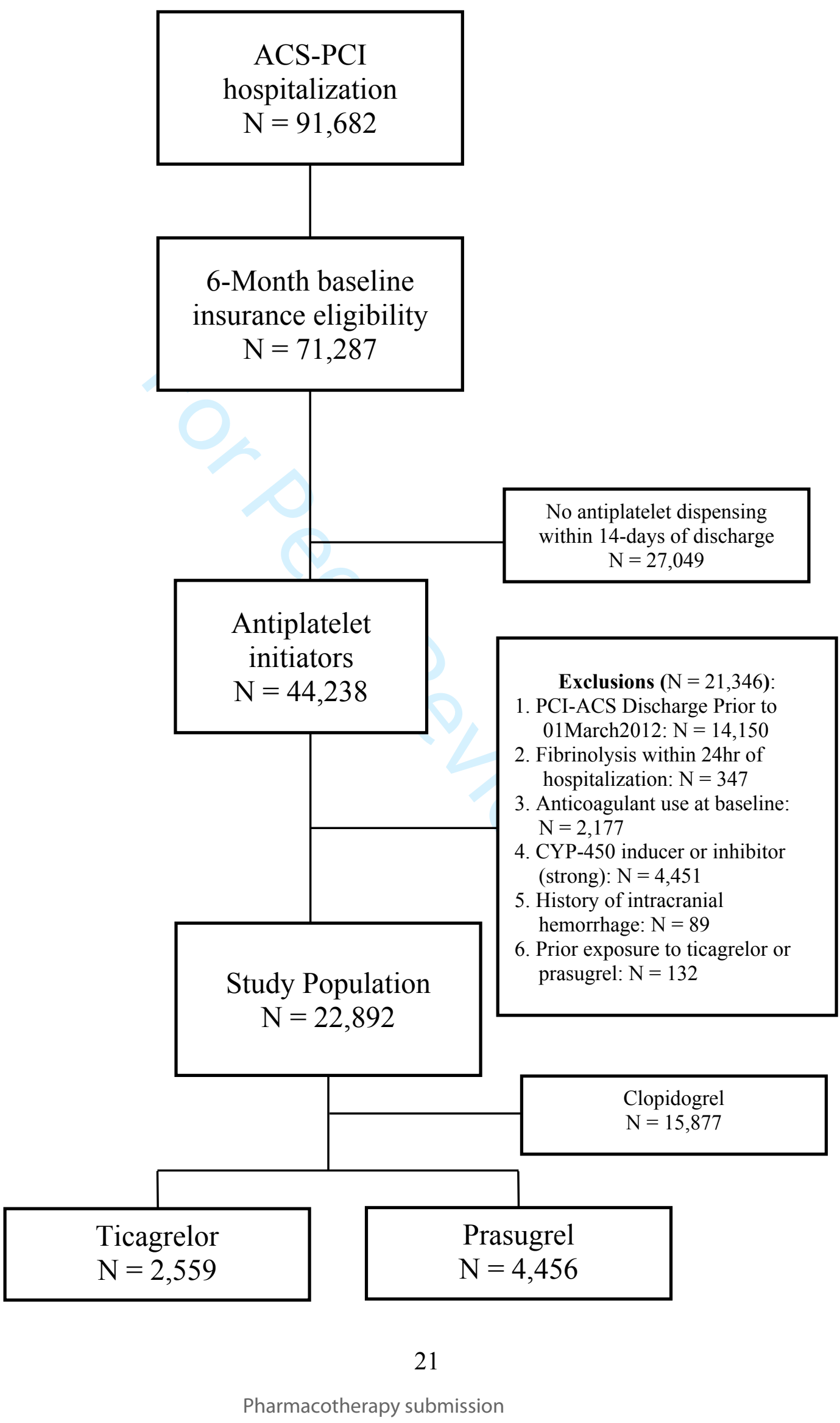


Table 1. Baseline Characteristics by Initial Exposure

\begin{tabular}{l|rc|rc|r}
\multicolumn{1}{c}{ Characteristics } & \multicolumn{2}{c}{ Ticagrelor (\%) } & \multicolumn{3}{c}{ Prasugrel (\%) } \\
& $\mathrm{n}=$ & 2,559 & $\mathrm{n}=$ & 4,456 & \multicolumn{1}{c}{$\mathbf{P}$} \\
\hline Age $( \pm$ SD) & 65.3 & \pm 11.7 & 59.8 & \pm 10.2 & $<0.01$ \\
Female & 820 & $(31)$ & 968 & $(21)$ & $<0.01$ \\
Tobacco Use & 633 & $(24)$ & 1,195 & $(26)$ & 0.04 \\
Hypertension & 2,041 & $(77)$ & 3,233 & $(71)$ & $<0.01$ \\
Hyperlipidemia & 1,916 & $(73)$ & 3,338 & $(73)$ & 0.64 \\
Carotid Artery Stenosis & 126 & $(5)$ & 143 & $(3)$ & $<0.01$ \\
Chronic Kidney Disease & 231 & $(9)$ & 282 & $(6)$ & $<0.01$ \\
Anemia & 277 & $(10)$ & 308 & $(7)$ & $<0.01$ \\
Chronic Obstructive & 386 & $(15)$ & 503 & $(11)$ & $<0.01$ \\
Pulmonary Disease & & & & & \\
Asthma & 133 & $(5)$ & 204 & $(4)$ & 0.27 \\
Percutaneous Transluminal & 271 & $(10)$ & 410 & $(9)$ & 0.07 \\
Coronary Angioplasty & & & & & \\
Coronary Artery Bypass & 136 & $(5)$ & 199 & $(4)$ & 0.12 \\
Graft & & & & & \\
Congestive Heart Failure & 514 & $(19)$ & 727 & $(16)$ & $<0.01$ \\
Atrial Fibrillation & 199 & $(8)$ & 232 & $(5)$ & $<0.01$ \\
Angiotensin-Converting & 1,027 & $(39)$ & 1,605 & $(35)$ & $<0.01$ \\
Enzyme/Angiotensin & & & & & \\
Receptor-Blocker & & & & & \\
Beta-Blocker & 778 & $(29)$ & 1,105 & $(24)$ & $<0.01$ \\
Diuretic & 425 & $(16)$ & 561 & $(12)$ & $<0.01$ \\
Statin & 999 & $(38)$ & 1,581 & $(35)$ & $<0.01$ \\
Diabetic Medication & 654 & $(25)$ & 1,058 & $(23)$ & 0.12 \\
Proton-Pump Inhibitor & 464 & $(18)$ & 646 & $(14)$ & $<0.01$ \\
Baseline Clopidogrel Exposure & 210 & $(8)$ & 278 & $(6)$ & $<0.01$
\end{tabular}


Table 2. Censoring Frequencies by Initial Exposure

\begin{tabular}{|c|c|c|c|c|c|}
\hline \multirow[b]{2}{*}{ Censoring Criterion } & \multicolumn{2}{|c|}{ Ticagrelor (\%) } & \multicolumn{2}{|c|}{ Prasugrel (\%) } & \multirow{2}{*}{$\begin{array}{c}\mathbf{P} \\
\text { Valu } \\
\mathbf{e}\end{array}$} \\
\hline & $\mathrm{n}=$ & 2,639 & $\mathrm{n}=$ & 4,566 & \\
\hline \multicolumn{6}{|l|}{ Days of follow-up } \\
\hline Mean $( \pm$ SD $)$ & 278 & $( \pm 113)$ & 294 & $( \pm 109)$ & \\
\hline Median (IQR) & 365 & $(190-365)$ & 365 & $(231-365)$ & \\
\hline $\begin{array}{l}\text { Insurance Disenrollment } \\
\text { During Follow-up }\end{array}$ & 1493 & $(57)$ & 2139 & $(47)$ & $<0.01$ \\
\hline Medication Switch & 931 & $(35)$ & 1278 & $(28)$ & $<0.01$ \\
\hline Switch to Clopidogrel & 815 & $(88)$ & 1273 & $(100)$ & \\
\hline Treatment Discontinuation & 230 & $(9)$ & 439 & $(10)$ & 0.22 \\
\hline
\end{tabular}


Table 3. Primary and Secondary Composite Outcome Frequencies by Initial Exposure. (Unadjusted)

\begin{tabular}{|c|c|c|c|c|c|}
\hline Outcomes & \multicolumn{2}{|c|}{$\begin{array}{c}\text { Ticagrelor }(\mathbf{\%}) \\
\mathrm{n}=2,639\end{array}$} & \multicolumn{2}{|c|}{$\begin{array}{l}\quad \text { Prasugrel (\%) } \\
\mathrm{n}=4,566\end{array}$} & $\begin{array}{c}\mathbf{P} \\
\text { value }\end{array}$ \\
\hline \multicolumn{6}{|l|}{ Primary Outcomes } \\
\hline All-cause Death & 33 & $(1.3)$ & 60 & $(1.3)$ & 0.82 \\
\hline Myocardial Infarction & 109 & $(4.1)$ & 150 & (3.3) & 0.06 \\
\hline Stroke & 29 & $(1.1)$ & 24 & $(0.5)$ & $<0.01$ \\
\hline Death, MI, Stroke & 119 & $(4.5)$ & 172 & (3.8) & 0.12 \\
\hline $\begin{array}{l}\text { Rate per } 100 \text { person-years } \\
\text { Secondary Outcomes }\end{array}$ & \multicolumn{2}{|c|}{7.1 per 100 person-years } & \multicolumn{2}{|c|}{5.3 per 100 person-years } & \\
\hline Gastrointestinal Bleed & 63 & $(2.4)$ & 84 & $(1.8)$ & 0.11 \\
\hline Other Major Bleed & 29 & $(1.1)$ & 41 & $(0.9)$ & 0.40 \\
\hline Intracranial Hemorrhage & 11 & $(0.4)$ & 17 & $(0.4)$ & 0.77 \\
\hline Bleeding Outcomes & 72 & $(2.7)$ & 98 & $(2.1)$ & 0.12 \\
\hline Rate per 100 person-years & \multicolumn{2}{|c|}{4.7 per 100 person-years } & \multicolumn{2}{|c|}{3.3 per 100 person-years } & \\
\hline
\end{tabular}


Table 4. Adjusted Hazard Ratios for Ischemic and Bleeding Events by Analysis Methods

\begin{tabular}{r|ccr} 
Time-Dependent Censor-Weighted (TD-CW) & HR & $\mathbf{9 5 \%}$ Cl & P \\
\hline All-cause death, MI, Stroke & 1.33 & $(1.04-1.69)$ & 0.02 \\
ICH, GI Bleed, Major Hemorrhage & 1.61 & $(1.19-2.17)$ & $<0.01$ \\
Intention-to-Treat (ITT) & & & \\
\hline All-cause death, MI, Stroke & 0.78 & $(0.58-1.06)$ & 0.11 \\
ICH, GI Bleed, Major Hemorrhage & 0.84 & $(0.58-1.20)$ & 0.33 \\
Clopidogrel-Naive TD-CW & & & \\
\hline All-cause death, MI, Stroke & 1.28 & $(0.99-1.66)$ & 0.06 \\
ICH, GI Bleed, Major Hemorrhage & 1.63 & $(1.19-2.23)$ & $<0.01$ \\
Clopidogrel-Naive ITT & & & \\
\hline All-cause death, MI, Stroke & 0.81 & $(0.59-1.11)$ & 0.19 \\
ICH, GI Bleed, Major Hemorrhage & 1.05 & $(0.73-1.51)$ & 0.79
\end{tabular}

Note: Myocardial infarction (MI), intracranial hemorrhage (ICH), gastrointestinal bleed (GI bleed) 
Figure 2. Estimated Survival Curve for death, myocardial infarction (MI), or stroke in weighted pseudopopulation

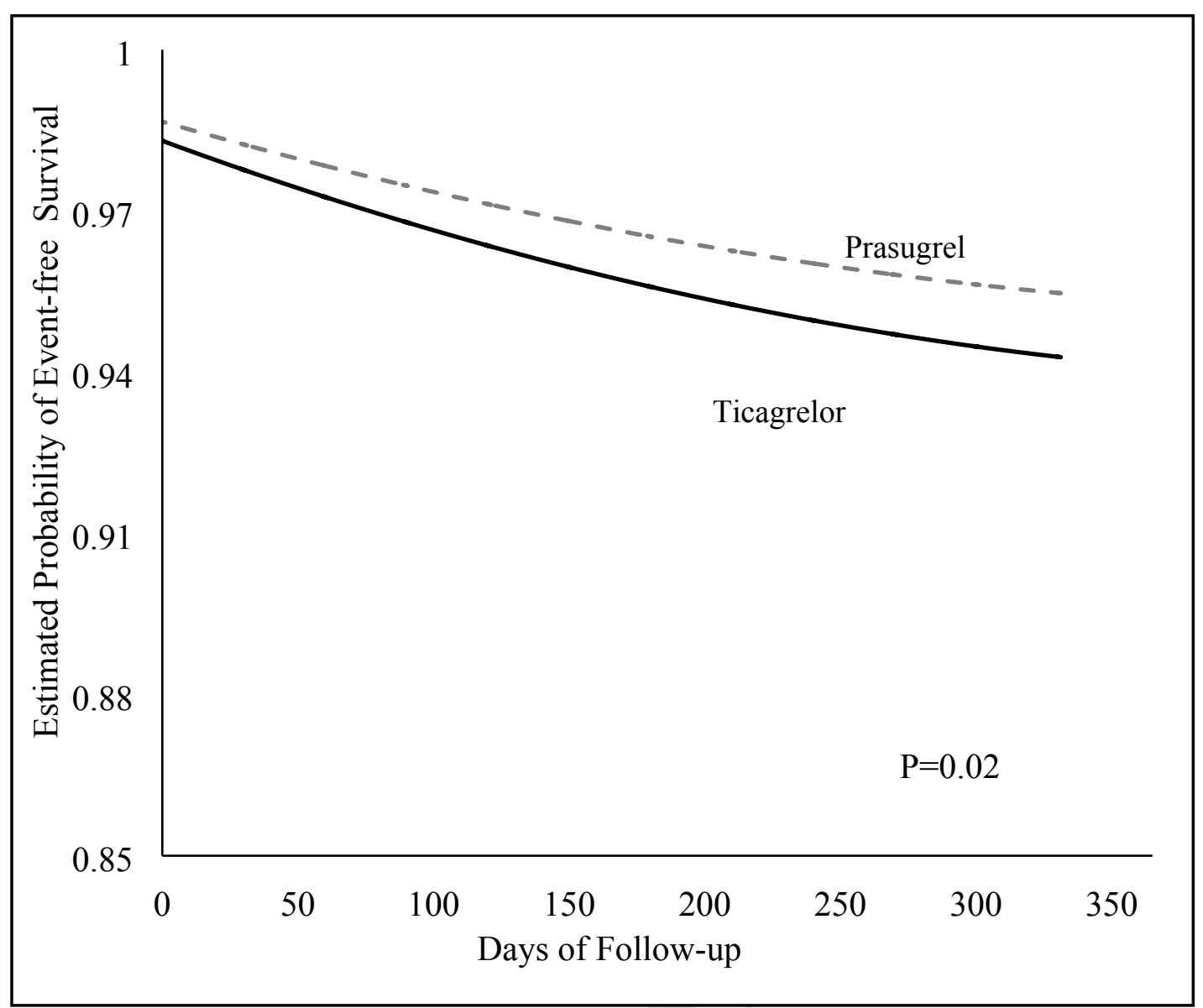


Figure 3. Estimated survival curve for gastrointestinal (GI) bleed, intracranial hemorrhage (ICH), or other major bleed requiring hospitalization in weighted pseudopopulation

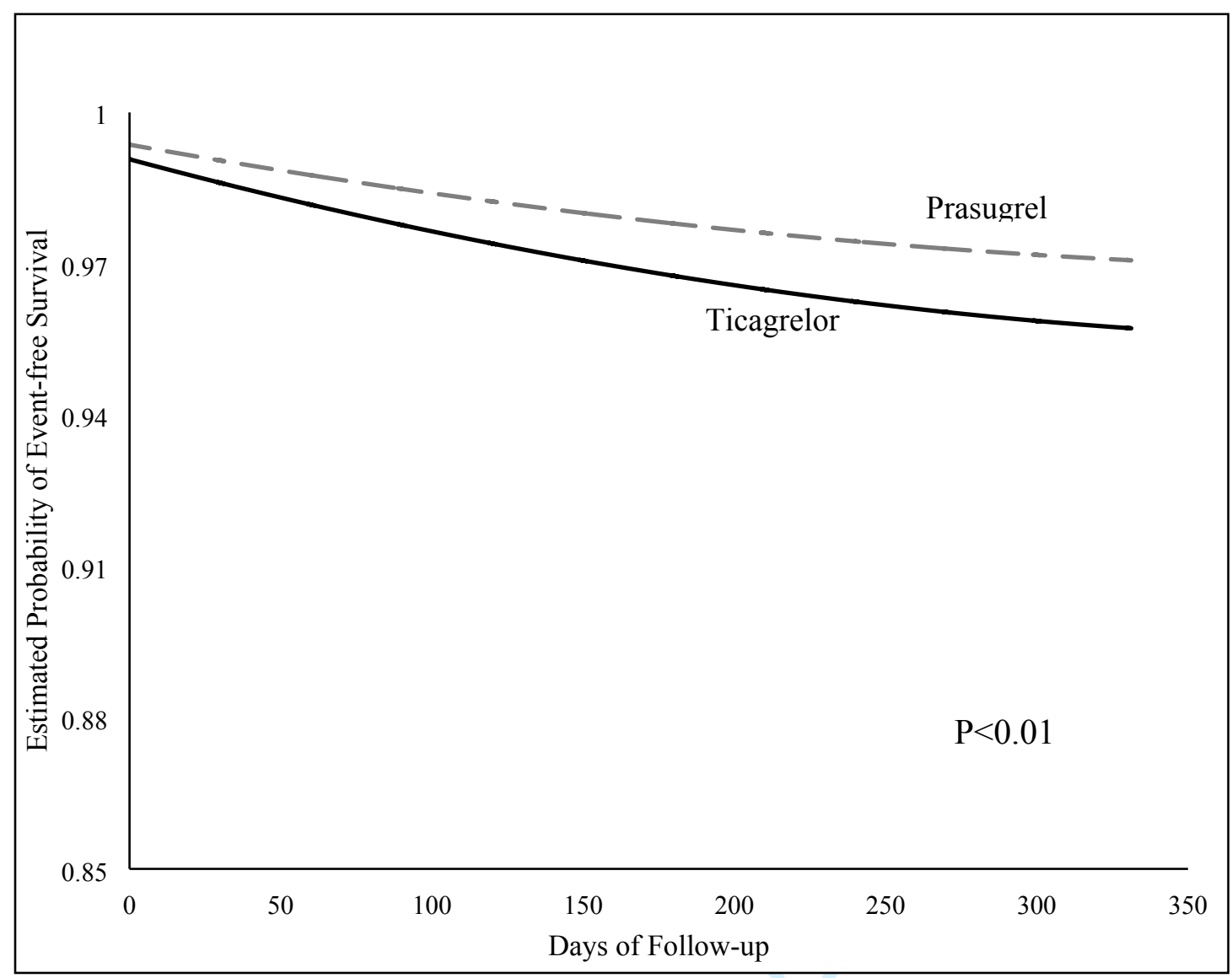


Appendix:

Appendix Table 1. Baseline Covariates IDC-9 Codes:

\begin{tabular}{|c|c|}
\hline Characteristic & $\begin{array}{l}\text { ICD-9 Codes for Baseline Characteristics } \\
\text { (decimal points removed) }\end{array}$ \\
\hline Percutaneous Coronary Intervention & $\begin{array}{l}360136023605360636073609006692980 \\
929819298292984929209292192924 \\
9292592928 \\
929339293492937929389294192943 \\
9294492973\end{array}$ \\
\hline Acute Coronary Syndrome & 410411 \\
\hline Fibrinolysis & $\begin{array}{l}37201372113721237213372143719592977 \\
9910\end{array}$ \\
\hline Tobacco & 3051 V1582 \\
\hline Hypertension & 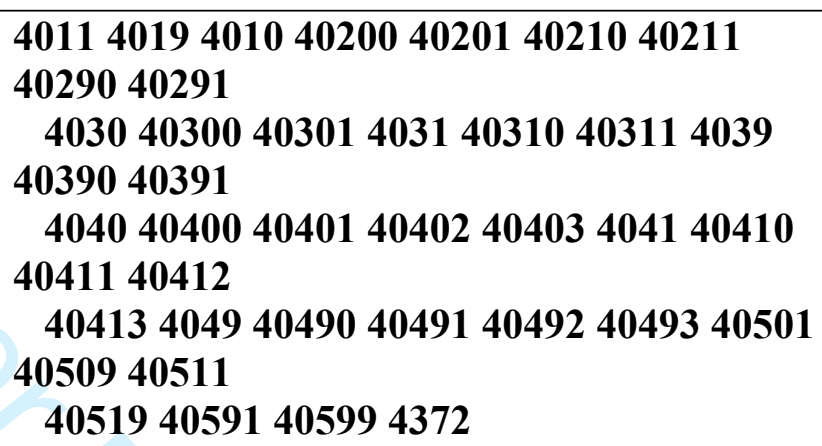 \\
\hline Hyperlipidemia & 27202721272227232724 \\
\hline Diabetes & 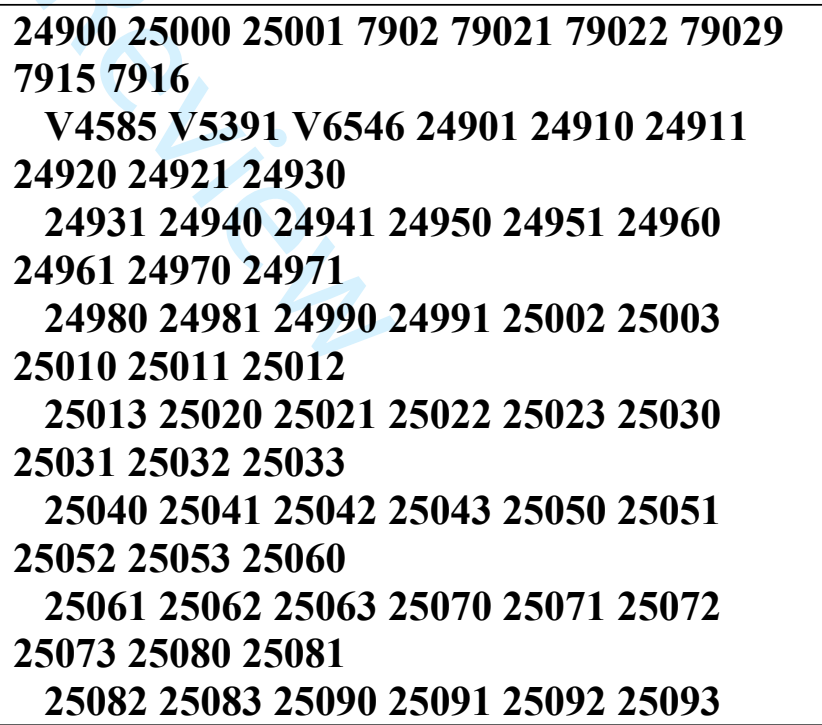 \\
\hline Carotid Artery Stenosis & 4331043311 \\
\hline Peripheral Vascular Disease & $\begin{array}{l}44004401440244020440214402244023 \\
440294404 \\
\quad 440844094439557055715579\end{array}$ \\
\hline End Stage Renal Disease & 5856 \\
\hline Chronic Kidney Disease & 585585158525853585458555859 \\
\hline
\end{tabular}




\begin{tabular}{|c|c|}
\hline Anemia & 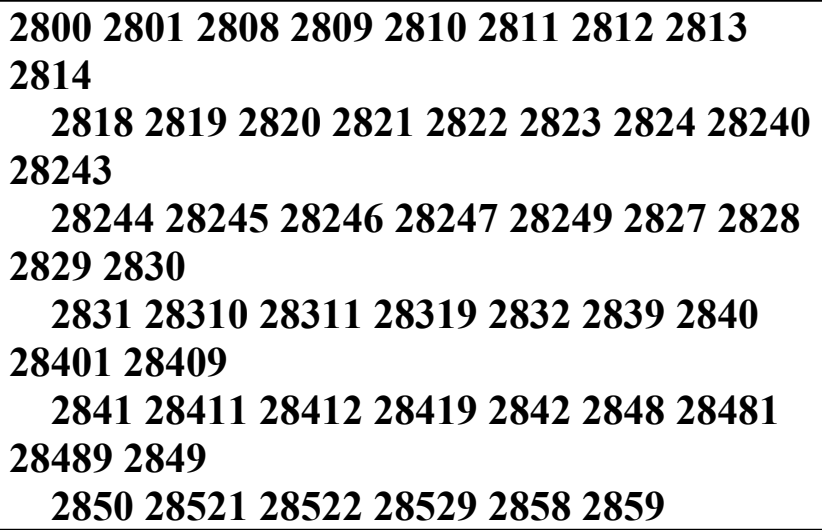 \\
\hline Chronic Obstructive Pulmonary Disease & $\begin{array}{l}4904910491149124912049121491224918 \\
49194920 \\
\quad 492849449404941496 \\
\end{array}$ \\
\hline Asthma & 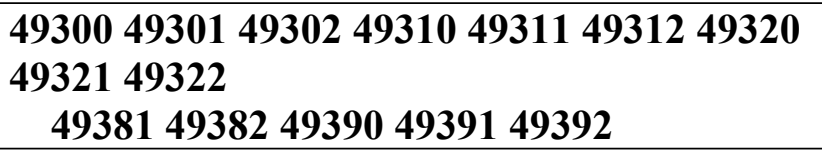 \\
\hline $\begin{array}{l}\text { Percutaneous Transluminal Coronary } \\
\text { Angioplasty }\end{array}$ & V4582 \\
\hline Coronary Artery Bypass Graft & V4581 \\
\hline Congestive Heart Failure & 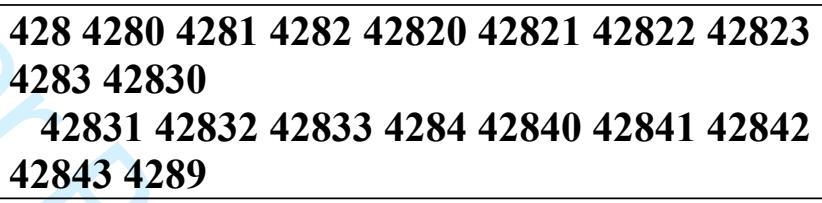 \\
\hline Atrial Fibrillation & 42731 \\
\hline Intracranial hemorrhage & 431430 \\
\hline
\end{tabular}


2

Appendix Table 2. Strong CYP-450 Inhibitors and Inducers

Strong

Inhibitors

Strong Inducers

CYP1A2

FluvoxaMINE

CYP2C8

Gemfibrozil

CYP2C19

\begin{tabular}{l|l}
\hline Fluconazole & $\begin{array}{l}\text { Apalutamide } \\
\text { RifAMPin }\end{array}$
\end{tabular}

CYP2D6

BuPROPion

Dacomitinib

FLUoxetine

PARoxetine

QuiNIDine

Tipranavir

\section{CYP2E1}

\begin{tabular}{l|l}
\hline Disulfiram & \\
&
\end{tabular}

CYP3A4

\begin{tabular}{l|l}
\hline Atazanavir & Apalutamide \\
Clarithromycin & CarBAMazepine \\
Cobicistat & Enzalutamide \\
Darunavir & Fosphenytoin \\
Idelalisib & Lumacaftor \\
Indinavir & Mitotane \\
Itraconazole & PHENobarbital \\
Ketoconazole & Phenytoin \\
(Systemic) & Primidone \\
Lopinavir & RifAMPin \\
MiFEPRIStone & \\
Nefazodone & \\
Nelfinavir & \\
Ombitasvir, & \\
Paritaprevir, and & \\
Ritonavir &
\end{tabular}


Ombitasvir,

Paritaprevir,

Ritonavir, and

Dasabuvir

Posaconazole

Ritonavir

Saquinavir

Telithromycin

Voriconazole 
Dear Dr Wen:

Manuscript ID 12-20-0746 entitled "Comparative Effectiveness and Safety of Prasugrel versus Ticagrelor following Percutaneous Coronary Intervention: A Real-World Data Study" which you submitted to Pharmacotherapy, has been reviewed. The comments of the reviewers are attached to this letter.

The reviewers have concerns with your paper, and major revisions would be needed to make it acceptable. Therefore, if you choose to make such changes to your paper and resubmit it, you must respond to the reviewers' comments and revise your manuscript accordingly. Your paper may be acceptable for publication provided you can satisfactorily respond to the reviewers' comments.

Please be mindful of word and reference counts when preparing your revision. You should refer to the author instructions for more information regarding word and citation limits.

Wiley Editing Services Available to All Authors Should you be interested, Wiley Editing Services offers expert help with manuscript, language, and format editing, along with other article preparation services. You can learn more about this service option at https://urldefense.proofpoint.com/v2/url?u=http-

3A www.wileyauthors.com eeo preparation\&d=DwIFaQ\&c=dWz0sRZOjEnYSN4E4J0dug\&r= 132BPCjAOTejTEebG8o5vFDgJj9BGfyfjtxESqsz3yc\&m= knkB6GLMGH6ImnMxWLsELuOsr U9fK5XtkzoAnTkk\&s=CPOjnlqCjxgcqx7wIVUzHBZo9ZtEiNkm7to5JQtD3Q\&e= . You can also check out Wiley's collection of free article preparation resources for general guidance about writing and preparing your manuscript at https://urldefense.proofpoint.com/v2/url?u=http-

3A www.wileyauthors.com eeo prepresources\&d=DwIFaQ\&c=dWz0sRZOjEnYSN4E4J0dug \&r=132BPCjAOTejTEebG8o5vFDgJj9BGfyfjtxESqsz3yc\&m= knkB6GLMGH6lmnMxWLsELuOsr U9fK5XtkzoAnTkk\&s=7h3hf8FNVGFKo0ZsLsJ_o iSlyEZ3M2VyNLUVFKN4c\&e= .

When submitting your revised manuscript, please upload a Word file that includes a copy of the reviewers' comments and your responses to these comments (highlighted). Please name this file "Response to Comments" and upload it as a "Supplementary" file. You can use this file to document any changes you make to the original manuscript. In order to expedite the processing of the revised manuscript, please be as specific as possible in your response to the reviewers' comments.

You will be unable to make your revisions on the originally submitted version of the manuscript. Instead, revise your manuscript using a word processing program and save it on your computer.

To submit your revised manuscript, log into https://urldefense.proofpoint.com/v2/url?u=http3A mc.manuscriptcentral.com pharmacotherapy\&d=DwIFaQ\&c=dWz0sRZOjEnYSN4E4J0du g\&r=132BPCjAOTejTEebG8o5vFDgJj9BGfyfjtxESqsz3yc\&m= knkB6GLMGH6Im- 
nMxWLsELuOsr U9fK5XtkzoAnTkk\&s=E7qP-3FGROSQNfOWy7WIsVyduR3Uto30WM5D4g3cAU\&e= and enter your Author Center, where you will find your manuscript title listed under "Manuscripts with Decisions." Under "Actions," click on "Create a Revision." Your manuscript number has been appended to denote a revision.

Once the revised manuscript is prepared, you can upload it and submit it through your Author Center.

I would very much appreciate it if you could upload your revision by 06-Mar-2021. If it is not possible for you to submit your revision in a reasonable amount of time (i.e., within 4 months) we may have to consider your paper as a new submission.

Once again, thank you for submitting your manuscript to Pharmacotherapy and I look forward to receiving your revision.

Cordially,

James Tisdale, PharmD

Scientific Editor, Pharmacotherapy

jtisdale@purdue.edu

Response: We sincerely appreciate the reviewer' constructive comments and editor's consideration. We will address all comments and resubmit the revised manuscript and point-to-point responses.

Reviewer(s)' Comments to Author:

Reviewer: 1

Comments to the Author

This manuscript is a real-world comparative effectiveness study of prasugrel and ticagrelor for ACS with $\mathrm{PCl}$.

1. Abstract: Please include a brief Background section.

Response: Added a background section to abstract

2. Page 8: Methods, Covariate Assessment - why wasn't STEMI, NSTEMI or unstable angina NOT included in the analysis? It is a predictor of outcome. If you were not able to discern from the database, describe in the limitations.

Response: Sub-codes differentiating between types of myocardial events such as STEMI or NSTEMI are not reliably utilized to differentiate as outcomes. ${ }^{1}$

3. Limitations Page 16: Exclusion Criteria/Figure 1: I calculated that $38 \%$ of patients in the database were excluded for lack of 14 -day dispensing which is capturing $72 \%$ not $83 \%$. Please reconcile. 
Response: Updated the language in the limitations section to make clearer. The 14-day window identified $83 \%$ of the patients within the database that had a pharmacy dispensing for the study treatment following PCI. During post-hoc analysis of the 27,049 patients excluded, only $10 \%$ had prescription dispensing for study agents at anytime following $\mathrm{PCl}$, with $3-5 \%$ having dispensings within 15 to 90 -days. Thus, using the 14-day window, we identified most of the patients with evidence of initiation of study treatment following PCI. Most of the 27,049 excluded did not have claims for the therapies of interest.

4. Figure 1: Where are those patients with PRIOR dispensing (page 7 line 15) in your figure? Response: There were 70, 27, and 35 patients in prasugrel, ticagrelor, and clopidogrel groups that had prior dispensings to either prasugrel or ticagrelor respectively. These patients were grouped with prior antiplatelet exclusion. Figure 1 has been updated to reflect this.

5. Exclusion Criteria/Figure 1: What were the strong CYP3A inducers and inhibitors prescribed? Figure 1 indicates $5-10 \%$ of patients were excluded. In practice those amount to clarithromycin and rifampin. Neither of which in my experience are prescribed often. Please re-evaluate these medications. Or did you mean moderate and strong?

Response: Patients taking strong CYP inducers or inhibitors were excluded (any CYP Isoenzyme, not just 3A4). A list is provided at the end of this document. The language was fixed in the manuscript.

6. Table 1: Please include whether the patient had STEMI, NSTEMI or unstable angina.

Response: STEMI and NSTEMI cannot be precisely assessed in the claims data. Sentence added in the limitations section.. No evidence has shown that STEMI, NSTEMI, or unstable angina significantly related to prescribing of two study drugs.

7. Table 3: You need to either define Composite Outcomes or change the labels. Consider redefining primary outcome as primary efficacy outcomes and secondary as secondary bleeding outcomes. As is, you have two labels: Composite Outcome that have different meanings.

Response: Edited table 3 so that outcomes are clearer.

8. All patients were followed for various times, correct? What was the median for each group? Add to the results. More importantly, why aren't the results presented in annualized events or per patient per some time period? (Tables 2 and 3 ) If it is taken into consideration in the analysis, please explain it more clearly in the methods.

Response: FU time is Added to table 2. Results as rate per person-time added to table 3. 
9. Figures 2 and 3 are not mentioned in the manuscript. Should they be? If not, delete.

Response: Sentence added at the end of the results section.

Reviewer: 2

Comments to the Author

General comments to the authors:

I would like to congratulate the authors on an interesting and timely manuscript. Overall, the paper is well written with some specific revisions discussed below. Here are some general comments on the manuscript:

1. The major issue with the manuscript is a thorough explanation of how and why their specific time exposure and censoring weighting methods impact the data findings. The data presented shows that it changes the interpretation of the data, but the reader does not gain an understanding of why and how the data changed. I would highly recommend more explanation of these methods, possible providing an example of how and why these methods change the findings in the introduction or discussion.

Response: Thank you for your important suggestion. We have added a sentence at the end of the second paragraph of the discussion section: "In order to replicate/mimic findings from the RCT, we need to conduct a study in which adherence is similar. To do this, we need to account for time-varying confounding and censoring to get an accurate 'on treatment' effect. Thus, we applied methods to account for time-dependent confounding, drug switching and discontinuation to account for differences in adherence/discontinuation between the real-world data and RCT. Accounting for these factors within the observational cohort resulted in dropout and treatment switching rates that were similar to the ISAR-REACT 5 trial. The final results from this analysis were in the same direction as the trial results We also have explained in detail the timedependent exposure and censoring weighting in the methods section.

2. Suggest changing the title to use "an observational study" instead of "a real-world data study". Although the use of real-world seems to be popular lately, especially by pharmaceutical manufacturers, these are plain and simply observational studies.

Response: changed to "Real-world data study".

3. Throughout the manuscript the "12" in P2Y12 should be subscript. Also, the correct term is acute coronary syndrome and not acute coronary syndromes. It is a single syndrome. It appears in both forms throughout the manuscript.

Response: Fixed both typos.

Specific comments for the authors: 
Page 5, lines 15-19: The statement about there not being a difference in bleeding in the PLATO trial is misleading. In practically all trials of P2Y12 inhibitors, the consistent definition of major bleeding is non-CABG TIMI major bleeding. This is what was used in TRITON-TIMI 38 and CURE. In PLATO the definition was manipulated to include all TIMI major bleeds. Since approximately $75 \%$ of patients going to CABG surgery have a major bleed, this dilutes the difference between the arms. When the consistent definition of non-CABG TIMI major bleeding is evaluated in PLATO, there is a significant increase compared to clopidogrel, with the same absolute increase as seen in TRITON. Both agents increase this consistent definition of major bleeding. Therefore, it would be appropriate to state that both agents reduce CV death, MI, and stroke by the same magnitude and both agent increase non-CABG TIMI major bleeding by the same magnitude.

Response: Changed the statement to "both agents reduce CV death, MI, and stroke by the same magnitude and both agents increase non-CABG TIMI major bleeding by the same magnitude."

Page 6, top: So there was no equipoise in the evaluation of your data? Then did you do a onetailed statistical analysis?

Response: In this observational study, there was no equipoise like there was in the RCT. Since as physicians perceived that Ticagrelor was superior to Prasugrel, there was confounding by indication which we attempted to account for through proper analysis. before ISAR-REACT 5 trial. ${ }^{2-6}$ The final estimates are adjusted HR, which is similar as the one-tailed statistical analysis that shows the higher risk in one agent.

Page 7, lines 10-13: It would seem more appropriate to include pharmacy claims within 30 or 45 days of discharge instead of 14 days. With many hospitals using meds-to-beds programs, the first month of medication is often provided to patients before they leave the hospital, and therefore, would not be captured in your data. Both ticagrelor and prausgrel also often gave the first month of medication for free, which would also have limited your ability to capture these patients. One of the main strengths of a comparative effectiveness study is the ability to capture larger numbers of patients than a randomized controlled trial. By limiting your pharmacy claims window, you reduce the power and impact of your results. You do mention this is your limitations, but seems to be an easy fix. It is someone confusing in your limitations discussion where you lose almost $25 \%$ of the study population because of this exclusion, but then state by changing the pharmacy claims date you did not gain many patients? These two statements do not seem to be consistent unless most of the patients did not have any claim until beyond 90 days.

Response: We sincerely appreciate reviewer's important suggestion. We actually have examined 30 days and 45 days and didn't observe a substantial increase in sample size. Also, patients may have events within 30 days or 45 days. The 14-day window identified $83 \%$ of the patients within the database that had a pharmacy dispensing for the study treatment following PCI. During post-hoc analysis of the 27,049 patients excluded, only about $10 \%$ had prescription dispensing for study agents at any time following $\mathrm{PCl}$, with $3-5 \%$ having dispensings within 15 to 90 -days. Thus, using the 14-day window, we identified most of the patients with evidence of initiation of study treatment following $\mathrm{PCl}$. Most of the 27,049 excluded did not have claims for the therapies of interest. 
Page 7, lines 38-43: The assigning of a random day in the month for each death seems odd. This reads as each death was given its own random day. It would seem more prudent to just give all deaths the same day of the month, such as the 1st, 15th, or 30th. This way is would be consistent for all deaths.

Response: We sincerely appreciate reviewer's insightful comments. We considered the random date assignment a more suitable approach than using a same mid-month date. We also measured day of death using the first and last day of the month and this did not change the study results.

Page 8, lines 22-24: Why were patients switched to only clopidogrel censored? Anytime a patients was switched, the data for that drug should end.

Response: Patients that switched to ticagrelor or prasugrel were not censored. The risktime attributed to the 'switched to' agent was included in estimating the pseudopopulation consistent with the time-varying exposure methods described in the statistical analysis section.

Page 8, line 42: $\quad$ Not sure why COPD (or asthma in your table) are included. They are not risk factors for CVD or bleeding.

Response: These baseline covariates have been included in other observational studies for these therapies.

Results: Please add the mean and median follow up times for each agent.

Response: Added to table 2.

Limitations: It would seem that the third limitation on the counting of subsequent events could be fixed by matching additional codes with hospitalizations and not clinic visits. As well requiring a primary code for ACS on subsequent admissions. This is actually critical to the internal validity of your data.

Response: From the reviewer's comments, we removed this limitation. The outcomes were identified via validated algorithms cited in the text evaluating hospitalizations and not clinic visits.

References: In Pharmacotherapy the journal names are not italicized and do not have a period after them. DOI numbers are also not used by the journal. Please be sure to review the instructions to authors before your submission. In references 3-5, 8, 9, 17, 18, 25, 28, and 29 all of the words in the title are capitalized, which is not appropriate for referencing. This inconsistency should have been caught by the lead or senior authors prior to submission.

Response: The references in the word document have no capitalizations. Perhaps this occurred during uploading or converting to PDF. I will check to be sure that this does not occur again. 
Table 2: $\quad$ Please add the actual event rates to this table in addition to the $\mathrm{HR}$ and $95 \% \mathrm{Cl}$. Also, the $\mathrm{HR}$ and $95 \% \mathrm{Cl}$ do not need to all be in separate columns. This is awkward to read. They can all go in the same column.

Response: Added event rates to table 3. Reformatted the columns in table 4.

Figure 2 and 3: Is it appropriate to label the y-axis "estimated probability of survival" when you are not evaluating survival. Figure 2 is the composite ischemic endpoints and Figure 3 is major bleeding. May be better to label as "estimated probability event free survival"

Response: Updated axis titles.

Reviewer: 3

Comments to the Author

Statistical and Methodological Review

This study uses contemporary pharmacoepidemiologic methods to overcome limitations of prior studies that have evaluated the comparative effectiveness and safety of prasugrel and ticagrelor. It has many strengths. I believe a few things need to be further discussed or specified and have some relatively minor concerns about other aspects of the methods.

Response: We sincerely appreciate the reviewer's positive comments. We will address all your comments diligently.

The time-period of the data is not entirely clear, and this is important because of the use of ICD9 codes to identify outcomes. The censoring criteria do not mention September 2015, the end of the study period, as a reason for censoring.

Response: Added Data end window in the 'censoring' section of methods.

Please comment on validation studies and the performance characteristics of the outcome definitions, i.e. PPV. I'm not entirely sure why 412, old MI, was included in that definition. 436 also is not a particularly valid code for stroke since guidance on stroke coding changed in the mid-2000s with instructions not to code strokes this way. The impact may be minimal since it is used less often, but using a study using recent data as a reference for its validity is important. It may also be useful to indicate the proportion of Mls and strokes identified with these more questionable codes. https://urldefense.proofpoint.com/v2/url?u=https3A www.ahajournals.org doi 10.1161 CIRCOUTCOMES.113.000371\&d=DwIFaQ\&c=dWz0s RZOjEnYSN4E4J0dug\&r=132BPCjAOTejTEebG8o5vFDgJj9BGfyfjtxESqsz3yc\&m= knkB6GLM GH6ImnMxWLsELuOsr U9fK5XtkzoAnTkk\&s=SMJCj78NXIIVHAcN0At4StZQpRPeUew1FeaOqWWeY $\underline{F g \& e}=$

Response: We evaluated outcomes as described in the cited validation algorithms. 
Please comment on the limitations of Social Security Death Index data for capturing mortality, and any validation studies vs. the National Death Index that may have been done in this data source. This data source has changed to no longer include state death data and sensitivity now appears to be limited.

Response: The death file included within this dataset is the Social Security Administration Death Master file. Since 2013 it was no longer mandatory for states to report death events to the Social Security Administration. As such, death events are expected to be non-differentially underreported. ${ }^{7}$ This is added to the limitations with the citation. ${ }^{6}$

Why is angina being treated as a time-varying covariate? It seems to me that it's likely on the causal pathway from drug choice to $\mathrm{Ml}$ since the purpose of these drugs is to prevent ischemic events. It seems reasonable to include in the analysis of bleeding since it may lead to other drugs that increase bleeding risk, but it's not clear to me why it would be considered a confounder with $\mathrm{MI}$ as an outcome.

Response: If a patient has an ACS, this will sometimes lead to a change in the agent used for DAPT (e.g. from prasugrel to ticagrelor).

A sensitivity analysis using only primary position MI codes may be beneficial for dealing with the challenges of differentiating visits related to prior $\mathrm{Ml}$ and new acute $\mathrm{MI}$.

Response: We utilized a validated claims algorithm for outcomes. This included MIrelated hospitalization with length of stay >= 3days. Since we are not evaluating MIrelated outpatient claims we believe that there is minimal opportunity for misclassifying historical MI event for a new MI event. We have removed this language from the limitations section.

The covariate list doesn't seem optimal for bleeding events, as it lacks peptic ulcer disease, inflammatory bowel disease, and some other GI abnormalities that pose bleeding risk. PPI use may handle peptic ulcer disease to some extent, but many people without peptic ulcer disease take PPIs. I'm not sure this necessitates redoing all the analyses, but can you comment on why these weren't included?

Response: We appreciate the reviewer's important suggestion. We acknowledge that these suggestions would improve the analysis. While many of the covariates were selected to mimic previous clinical trials, information regarding gastrointestinal disorders or abnormalities may be factors of interest.

Please include an appendix that specifies the ICD codes used to define different covariates, as this is necessary for reproducibility of the work and assessment of the appropriateness of the methods.

Response: Thank you for your important suggestion. We have added an eTable that present all ICD codes to define the covariates. 


\section{Citations:}

1. Alexandrescu, R., Bottle, A., Jarman, B. \& Aylin, P. Current ICD10 codes are insufficient to clearly distinguish acute myocardial infarction type: a descriptive study. BMC Health Serv Res 13, 468 (2013).

2. Song, C. et al. Ninety-Day Readmission and Long-Term Mortality in Medicare Patients ( $\geq 65$ Years) Treated With Ticagrelor Versus Prasugrel After Percutaneous Coronary Intervention (from the Blue Cross Blue Shield of Michigan Cardiovascular Consortium). Am. J. Cardiol. 120, 1926-1932 (2017).

3. Coons, J. C. et al. Comparative Effectiveness and Safety Analysis of Dual Antiplatelet Therapies Within an Integrated Delivery System. Ann Pharmacother 51, 649-655 (2017).

4. Yudi, M. B. et al. Clopidogrel, prasugrel or ticagrelor in patients with acute coronary syndromes undergoing percutaneous coronary intervention. Intern Med J 46, 559-565 (2016).

5. Alexopoulos, D. et al. Contemporary antiplatelet treatment in acute coronary syndrome patients undergoing percutaneous coronary intervention: 1-year outcomes from the GReek AntiPlatElet (GRAPE) Registry. J. Thromb. Haemost. 14, 1146-1154 (2016).

6. Kim, K. et al. Comparative Effectiveness of Oral Antiplatelet Agents in Patients with Acute Coronary Syndrome. Pharmacotherapy 37, 877-887 (2017).

7. Levin, M. A., Lin, H.-M., Prabhakar, G., McCormick, P. J. \& Egorova, N. N. Alive or dead: Validity of the Social Security Administration Death Master File after 2011. Health Services Research 54, 24-33 (2019). 
Appendix:

Strong CYP-450 inducers and inhibitors

\begin{tabular}{|c|c|}
\hline $\begin{array}{l}\text { Strong } \\
\text { Inhibitors }\end{array}$ & $\begin{array}{l}\text { Strong } \\
\text { Inducers }\end{array}$ \\
\hline \multicolumn{2}{|l|}{ CYP1A2 } \\
\hline \multicolumn{2}{|l|}{ FluvoxaMINE } \\
\hline \multicolumn{2}{|l|}{ CYP2C8 } \\
\hline \multicolumn{2}{|l|}{ Gemfibrozil } \\
\hline \multicolumn{2}{|l|}{ CYP2C19 } \\
\hline \multirow[t]{2}{*}{ Fluconazole } & Apalutamide \\
\hline & RifAMPin \\
\hline \multicolumn{2}{|l|}{ CYP2D6 } \\
\hline \multicolumn{2}{|l|}{ BuPROPion } \\
\hline \multicolumn{2}{|l|}{ Dacomitinib } \\
\hline \multicolumn{2}{|l|}{ FLUoxetine } \\
\hline \multicolumn{2}{|l|}{ PARoxetine } \\
\hline \multicolumn{2}{|l|}{ QuiNIDine } \\
\hline \multicolumn{2}{|l|}{ Tipranavir } \\
\hline \multicolumn{2}{|l|}{ CYP2E1 } \\
\hline \multicolumn{2}{|l|}{ Disulfiram } \\
\hline \multicolumn{2}{|l|}{ CYP3A4 } \\
\hline Atazanavir & Apalutamide \\
\hline Clarithromycin & CarBAMazepine \\
\hline Cobicistat & Enzalutamide \\
\hline Darunavir & Fosphenytoin \\
\hline Idelalisib & Lumacaftor \\
\hline Indinavir & Mitotane \\
\hline Itraconazole & PHENobarbital \\
\hline $\begin{array}{l}\text { Ketoconazole } \\
\text { (Systemic) }\end{array}$ & Phenytoin \\
\hline Lopinavir & Primidone \\
\hline MiFEPRIStone & RifAMPin \\
\hline Nefazodone & \\
\hline Nelfinavir & \\
\hline
\end{tabular}




\begin{tabular}{|l|l|}
\hline $\begin{array}{l}\text { Ombitasvir, } \\
\text { Paritaprevir, } \\
\text { and Ritonavir }\end{array}$ & \\
\hline $\begin{array}{l}\text { Ombitasvir, } \\
\text { Paritaprevir, } \\
\text { Ritonavir, and } \\
\text { Dasabuvir }\end{array}$ & \\
\hline Posaconazole & \\
\hline Ritonavir & \\
\hline Saquinavir & \\
\hline Telithromycin & \\
\hline Voriconazole & \\
\hline
\end{tabular}

